\title{
Constrictive pericarditis as a complication of coronary artery bypass surgery
}

\author{
PAULO RIBEIRO, RALPH SAPSFORD, TOM EVANS, ^ GEORGE PARCHARIDIS, $†$ \\ CELIA OAKLEY \\ From the Department of Clinical Cardiology and Cardiothoracic Surgery, Hammersmith Hospital, London
}

SUMMARY Although it is now recognised as a rare complication of cardiac surgery, constrictive pericarditis was diagnosed in three patients after coronary artery bypass surgery. The time interval between cardiac surgery and the development of constrictive features varied from two to six weeks. All three patients presented with severe congestive heart failure. Haemodynamic findings were characteristic of constrictive pericarditis. Pericardial thickening detected by computed tomography in one patient was useful in establishing a definite diagnosis. One of the patients had a serous constrictive effusive pericarditis, and surgical pericardial drainage was needed. The other patient underwent pericardiectomy with preservation of the grafts. The diagnosis of constrictive pericarditis should be considered in patients presenting with unexplained right sided heart failure after cardiac surgery.

Constrictive pericarditis is an uncommon disease but an important one to recognise as it is potentially curable. Bacterial infection of the pericardium after cardiac surgery is a rare but recognised cause of constrictive pericarditis, ${ }^{1}$ but the development of constriction without overt preceding infection has only recently been reported. ${ }^{2-5}$

Constrictive pericarditis can be induced experimentally by trauma and by injection of autologous blood in the pericardial sac. ${ }^{6}$ Traumatic haemopericardium with subsequent development of constrictive pericarditis has been reported. ${ }^{7}$ It is surprising that constrictive pericarditis does not complicate cardiac surgery more often.

We report the clinical presentation, diagnosis, and management of three patients who developed constrictive pericarditis as a complication of coronary artery bypass surgery.

^Present address: Royal Free Hospital, Pond Street, Hampstead, London NW3. †Present address: University of Thessaloniki, 82 Egnatia Street, Greece.

Requests for reprints to Dr C M Oakley, Hammersmith Hospital, Royal Postgraduate Medical School, Du Cane Road, London W12 OHS.

Accepted for publication 27 October 1983

\section{Case reports}

CASE 1

A 67 year old Greek man gave a three year history of typical exertional angina. He was otherwise in good health. There was a history of hypertension, and he had diabetes mellitus controlled by glibenclamide.

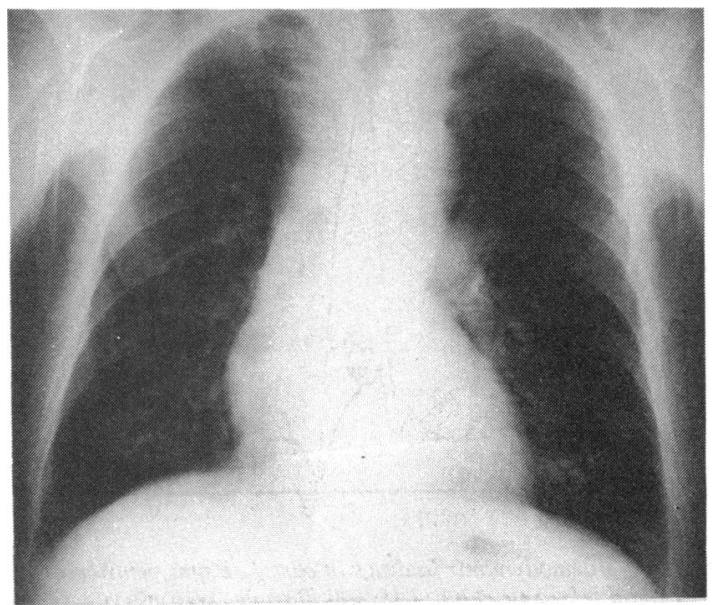

Fig. 1 Chest $x$ ray film of case 1 before pericardectomy showing mild cardiomegaly and clear lung fields. 
Physical examination was unremarkable, and both the electrocardiogram and chest $x$ ray examinations showed no abnormality (Fig. 1). Cardiac catheterisation showed good left ventricular function with inferior hypokinesia. Coronary angiography detected a normal left main stem and three vessel disease. In March 1981 he had saphenous bypass grafts placed in the left anterior descending, the marginal branch of the circumflex, and the right coronary arteries. The pericardium was left open. His postoperative course was uneventful, but in retrospect his electrocardiogram on discharge showed low voltage and poor progression of the $\mathrm{R}$ wave. Chest $x$ ray examination showed a normal heart size with a left pleural effusion. Six weeks later in Greece he started to complain of tiredness, breathlessness, and ankle swelling. He was apyrexial, and a pericardial rub was heard. Chest $x$ ray films showed cardiomegaly. Postcardiotomy syndrome was diagnosed. He was given diuretics and steroids and his condition improved. His heart size decreased on chest $x$ ray examination (Fig. 1), but his symptoms recurred.

Six months after the operation he was referred back to London. On physical examination his venous pressure was $15 \mathrm{~cm}$ with an $M$ shaped contour. There was no pulsus paradoxicus, and an early third heart sound was heard. His chest $x$ ray film was clear, and
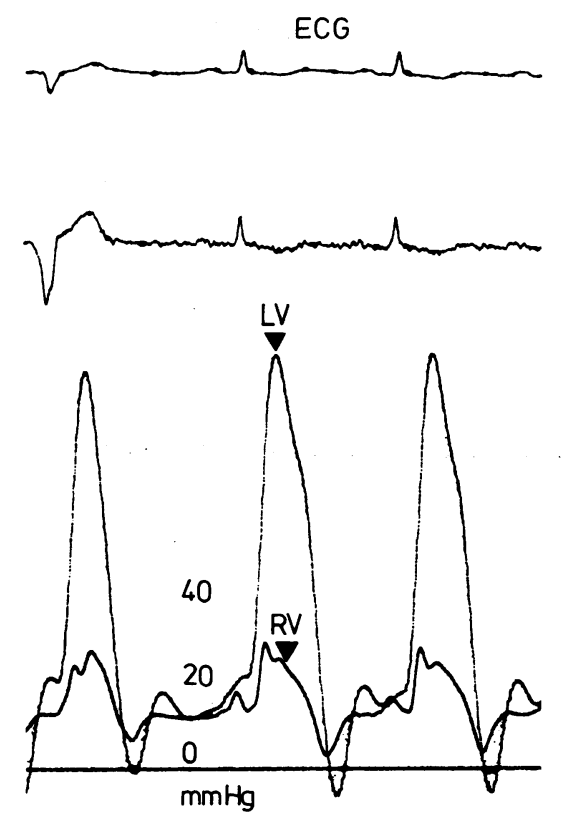

Fig. 2 Haemodynamic findings in case 1. Right ventricular $(R V)$ and left ventricular $(L V)$ end diastolic pressures are similarly raised with a dip-plateau contour. Note the early dip approaching the zero level ( $\mathrm{mm} \mathrm{Hg}$ ). his liver was palpable $4 \mathrm{~cm}$ below the costal margin. There was ascites and ankle swelling. An electrocardiogram showed sinus rhythm with low voltage. Cardiac catheterisation showed a very small vigorously contracting left ventricle and no change in the native coronary anatomy. There were three patent grafts. A right ventricular angiogram was normal. The haemodynamic measurements were typical of constriction with similar high diastolic pressures in both ventricles. An early dip and plateau were seen in the pressure tracings of both ventricles (Fig. 2). At pericardiectomy a mass of dense fibrous tissue filled the anterior mediastinum and was adherent to the pericardium. The anterior and lateral surfaces of the pericardium were excised in between the grafts, which were not disturbed. His symptoms and constrictive features resolved slowly but completely over the following six months.

\section{CASE 2}

A 55 year old man sustained an anterior myocardial infarct in April 1982. Since then he complained of typical exertional angina. He smoked 50 cigarettes a day. There was no history of hypertension. Diabetes mellitus was diagnosed in 1981 and controlled with oral hypoglycaemic agents. He was admitted for investigation in December 1982. Physical examination was unremarkable. An electrocardiogram showed sinus rhythm and signs of an old inferior myocardial infarction. Chest $x$ ray films were normal. Left ventricular angiography showed inferior dyskinesia with overall good function. Coronary angiogram showed a left main stem stricture and three vessel disease. Coronary bypass graft surgery was performed a month after admission; four saphenous grafts were inserted. His postoperative course was uneventful, and he was discharged 10 days later taking aspirin and dipyridamole. At the time his electrocardiogram showed minor ST-T changes and slightly lower voltage than before the operation. Chest $x$ ray examination showed the heart to be at the upper limit of normal size.

A month later he complained of sickness, tiredness, breathlessness, and ankle swelling over the previous two weeks. On physical examination he was apyrexial, O and the venous pressure was $15 \mathrm{~cm}$ with pulsus paradoxicus of $15 \mathrm{~mm} \mathrm{Hg}$. His chest $x$ ray film was clear. Auscultation detected an early third heart sound. There was gross ascites and ankle swelling. An electrocardiogram showed very low voltage. Chest $x$ ray examination indicated slight cardiomegaly with bilat- $\stackrel{0}{\rightarrow}$ eral pleural effusions. At cardiac catheterisation $T$ haemodynamic measurements were typical of constriction (Fig. 3). The left ventricular angiogram $\mathbb{D}$ showed a small vigorously contracting chamber. The $\mathbb{D}$ native coronary arteries were unchanged. There were 


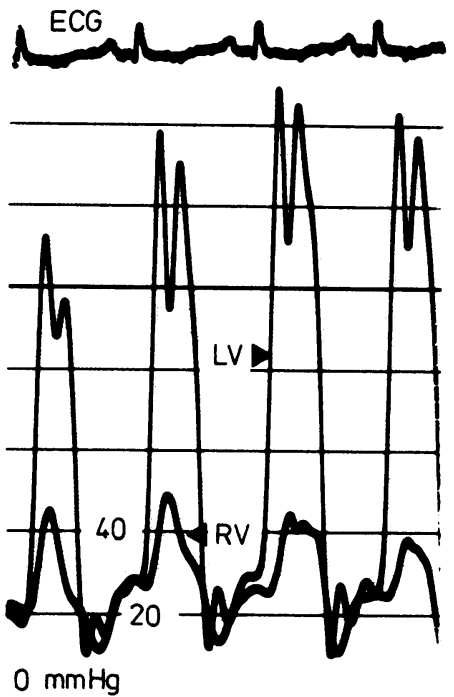

Fig. 3 Haemodynamic findings in case 2. Right ventricular $(R V)$ and left ventricular $(L V)$ end diastolic pressures are similarly raised. The early dip does not approach the zero level ( $\mathrm{mm} \mathrm{Hg}$ ).

three patent grafts. Echocardiography showed a pericardial effusion. Attempted pericardial tap was unsuccessful. He was referred for radical pericardectomy. At operation the pericardium was thickened, and severe adhesions to the mediastinum and sternum were found. A pericardial effusion with blood clots was drained. Because of dense adhesions between the four grafts and the pericardium an attempt at radical pericardiectomy was abandoned. During the following day the gross ascites and ankle swelling subsided. Ten days later his condition was considerably improved. An electrocardiogram still showed low voltage. Two months later $\mathrm{M}$ mode and cross sectional echocardiography showed pericardial thickening (Fig. 4).

\section{CASE 3}

A 47 year old man had a history of myocardial infarction complicated by ventricular fibrillation in 1980 . Since then he had exertional angina. He smoked 12 cigarettes a day. He was admitted for investigation in June 1982. Physical examination showed no abnormalities. Electrocardiogram showed signs of old inferior and anterior myocardial infarction. A heart size at the upper limit of normal and clear lung fields were evident on the chest $x$ ray films. Cardiac catheterisation showed overall good left ventricular function, but there was anteroapical dyskinesia. Coronary angiography indicated two vessel disease. He was referred for coronary bypass surgery and had two saphenous grafts three days after admission. His postoperative recovery was uneventful. At discharge, his electrocardiogram showed minor ST-T changes and lower voltage than before the operation. The heart shadow was unchanged on $x$ ray examination.

A month later when seen as an outpatient he complained of feeling that his head and neck were congested and of tiredness, breathlessness, and ankle swelling during the last few days. On physical examination his venous pressure was $8 \mathrm{~cm}$ with an $M$ shaped pulse contour. His chest was clear, and heart sounds were normal. There was pitting oedema over both ankles. An electrocardiogram showed low voltage. On chest $x$ ray examination the heart size was unchanged, but small bilateral pleural effusions were seen. Ventilation perfusion scan, superior vena cavagram, and left leg venogram were normal. Echocardiogram showed no pericardial effusion. Computed tomography of the thorax indicated pericardial calcification and thickening (Fig. 5). At cardiac catheterisation the right ventricular angiogram showed a small, but well contracting chamber. Right sided haemodynamic measurements were characteristic of constriction. Constrictive pericarditis after cardiac surgery was diagnosed. As the patient was unwilling to undergo further surgery it was decided to defer pericardiectomy. He was seen three months later when his symptoms and clinical features were unchanged.
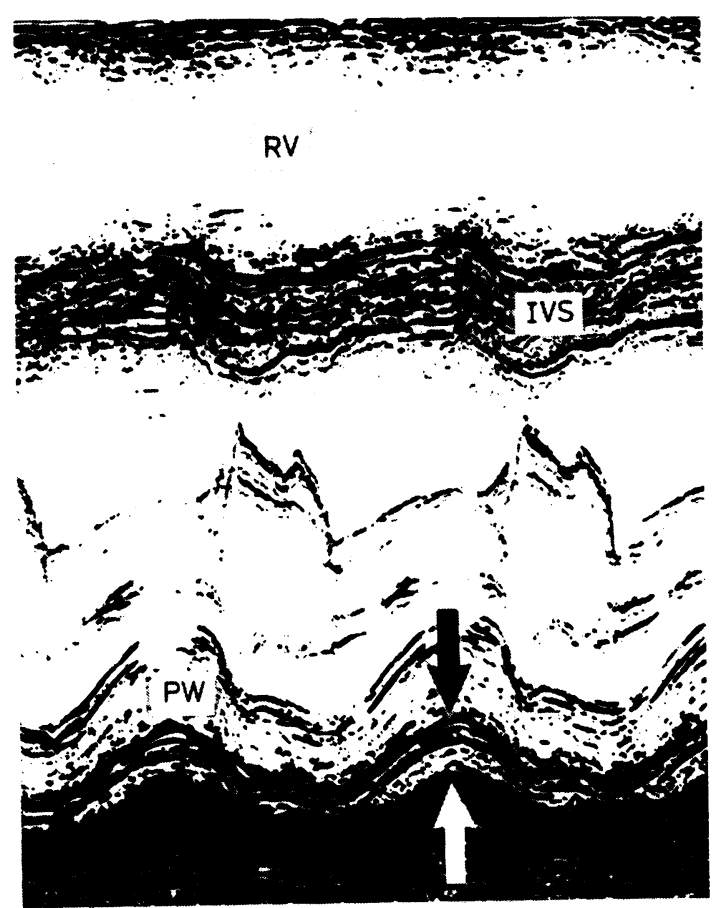

Fig. $4 M$ mode echocardiogram of the left ventricle in case 2 showing parallel motion of the visceral and parietal pericardial layers, implying that they are adherent (arrows). PW, posterior wall; IVS, interventricular septum; $R V$, right ventricle. 

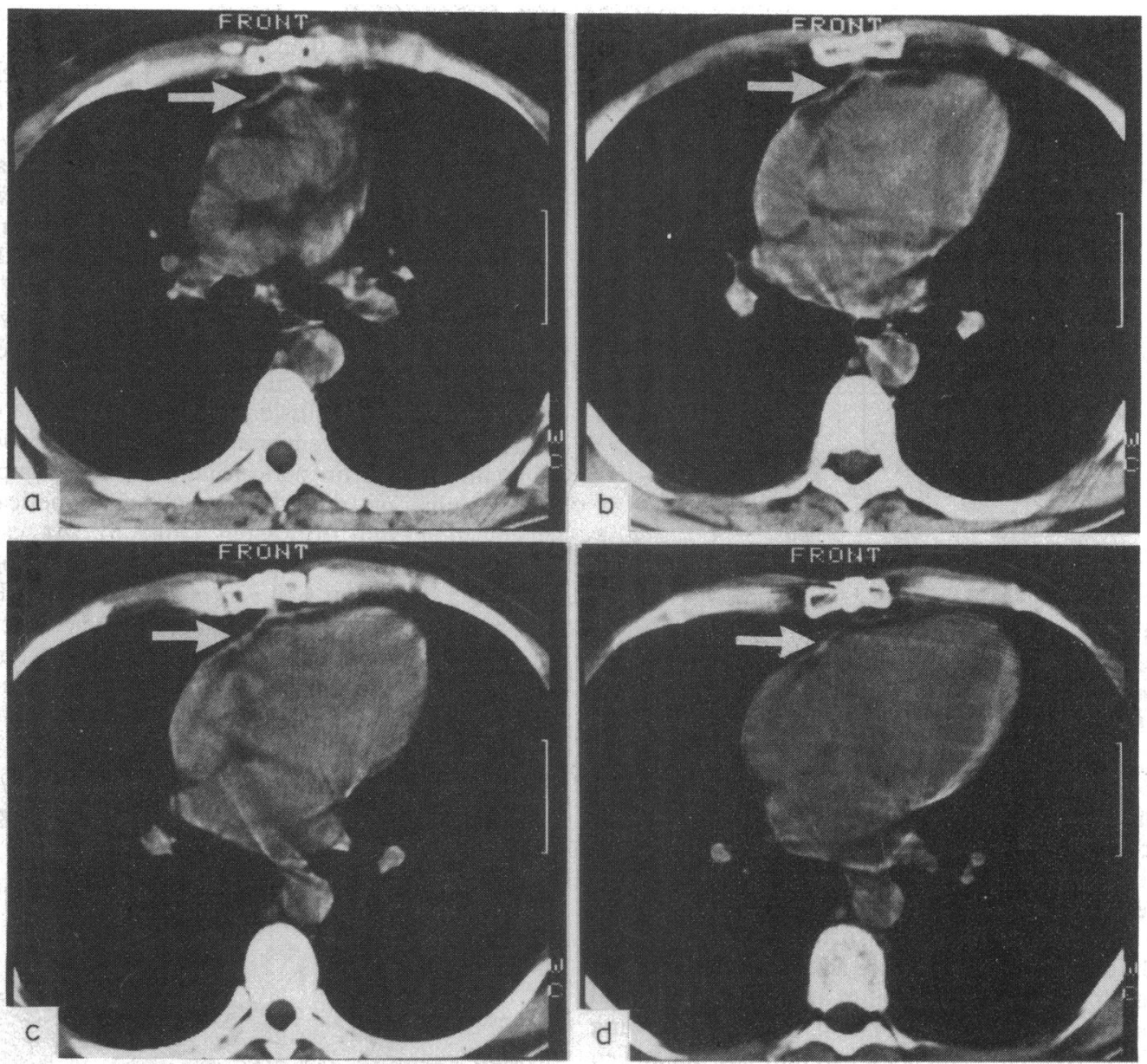

Fig. 5 Serial chest computed tomograms (case 3) showing extensive pericardial calcification (arrows).

\section{Discussion}

Constrictive pericarditis as a consequence of cardiac surgery was first recognised at necropsy ${ }^{8}$ and seems to be a surprisingly rare complication of cardiac surgery. A few cases have been reported after coronary artery bypass surgery, 235 prosthetic valve replacement, 59 and atrial septal repair. ${ }^{10}$ The diagnosis should be suspected in patients presenting with right sided heart failure after operation, even if there is associated left ventricular failure. ${ }^{2}$

One of the reasons for the late emergence of constriction as a complication of cardiac surgery is the difficulty in recognising it in patients who have had surgery for congenital or valvular heart disease and who often have other reasons for raised venous filling pressures. The symptoms and clinical features in our patients, who had only coronary disease, were typical of constrictive pericarditis. ${ }^{11}$ The pathological findings varied from constrictive effusive pericarditis in the patient (case 1) with pericardial thickening, adhesions, and a pericardial effusion containing blood clots to those of established chronic constrictive pericarditis as described by Paul Wood. ${ }^{12}$

The main differential diagnosis in patients presenting with signs of constriction early after cardiac $\mathscr{Q}$ surgery is between constrictive effusive pericarditis 0 and late cardiac tamponade. Haemodynamic studies in serous effusive constrictive pericarditis show a $\stackrel{\mathcal{C}}{\rightarrow}$ prominent dip plateau ventricular pressure. ${ }^{13}$ The $T$ early dip does not approach the zero level $(\mathrm{mm} \mathrm{Hg})$ as in chronic constrictive pericarditis as a result of the effusive element. ${ }^{1314}$ In cardiac tamponade the early $\stackrel{\mathbb{Q}}{\mathcal{Q}}$ diastolic dip is absent. ${ }^{1415}$ This is explained by the 
tense effusion that prevents filling of the ventricles from early diastole. Furthermore, the venous pressure wave form in serous effusive constrictive pericarditis shows equal systolic and diastolic descents (xy), ${ }^{13}$ whereas in cardiac tamponade the $\mathrm{y}$ descent is absent. ${ }^{1315}$ Computed tomography is diagnostic in showing pericardial thickening or effusion as well as their degree and location. In addition these scans show the haemodynamic consequences with a small narrow left ventricle with a sinusoidal septal contour when constriction is severe. ${ }^{16}$ Perioperative right ventricular infarction should also be considered. The characteristic haemodynamic findings, right ventricular angiography or MUGA scan, or cross sectional echocardiography will exclude it. 1718

The pathogenesis of postoperative pericardial constriction and the reasons for its rarity are not fully understood. Residual blood elements within the pericardial sac, especially in those areas of major involvement, have been found at cardiac surgery in most patients with chronic constrictive pericarditis of various aetiologies. ${ }^{19}$ Even more important are Cohen and Greenberg's findings of organised haematoma, loculated clots, and unclotted viscous blood during pericardectomy within the pericardial space in patients with constrictive pericarditis after cardiac surgery. ${ }^{9}$ Injection of autologous blood into the pericardial cavity failed to produce constrictive pericarditis in animals. Nevertheless, when the pericardium was temporarily dried and blood was injected into the pericardium of rabbits severe adhesions between pericardium and epicardium developed. ${ }^{20}$ Furthermore, in dogs haemopericardium as a result of trauma was followed by clinical and pathological evidence of constrictive pericarditis. ${ }^{6}$ This suggests that blood and trauma may be important factors in the pathophysiology of constrictive pericarditis after cardiac surgery. In humans, constrictive pericarditis after traumatic haemopericardium has been well documented. ${ }^{21} 22$ To support this concept, there is experimental evidence of decreased pericardial mesothelial fibrinolytic activity after mechanical injury. ${ }^{23}$ Alternatively, a clinically undetectable low grade infection may be a contributive factor. A role for the postcardiotomy syndrome in the pathogenesis of constriction is another possibility. The high incidence of this syndrome ${ }^{24}$ however, makes it difficult to establish a link with the rare occurrence of constrictive pericarditis after cardiac surgery.

Different surgical techniques are used to avoid fluid accumulation within the pericardium. In our institution the pericardium is left open to permit drainage into the pleural spaces and mediastinum. Furthermore, a pericardium not properly protected during surgery will shrink and if closed may lead to constric- tion. ${ }^{25}$ Even when the pericardium is not closed constrictive pericarditis may develop. ${ }^{9}$ Douglas et al found frequent blood clots around the heart when the pericardium was left open in those cases in which reoperation was necessary for excessive bleeding. ${ }^{26}$ Most postoperative bleeding originates from structures outside the pericardium. ${ }^{25}$ This would favour closing the pericardium. Cunningham et al in their series found no intrapericardial clot when reoperation for excessive bleeding was performed when the pericardium was closed. ${ }^{25}$ Also, far fewer pericardial effusions were present when the pericardium was left closed. ${ }^{25}$

Pericardial irrigation with povidone-iodine solution has been implicated as a contributory factor in the pathogenesis of postoperative constrictive pericarditis. ${ }^{2}$ None of our patients was exposed to this solution. The available experimental evidence and the absence of adhesions after peritoneal irrigation make it unlikely. ${ }^{2}$ Interestingly, constrictive pericarditis has been reported after pericardial irrigation with Dakin's solution. ${ }^{27}$

Medical treatment with diuretics and steroids has been successful in resolving constrictive signs in two instances after cardiac surgery, ${ }^{5}$ probably by facilitating reabsorption of pericardial fluid and thus reversing the effusive element in serous effusive constrictive pericarditis. Constrictive features were relieved by surgical drainage in one of our patients; radical pericardectomy may be needed in the future. Saphenous vein grafts have been nicked during attempts at pericardiectomy. ${ }^{5}$ Our patient had three patent grafts with dense adhesions to the pericardium. Attempts at radical pericardiectomy were abandoned.

Radical pericardiectomy is the treatment of choice in constrictive pericarditis. It entails removal of virtually the entire parietal pericardium from all cardiac surfaces. Delayed recovery after partial pericardiectomy has been attributed to myocardial atrophy during long periods of constriction. ${ }^{28}$ Somerville suggested that inadequate decortication was responsible for the delayed improvement and persistence of constrictive symptoms. ${ }^{28}$ This concept has been supported by the report of eight successful reoperations for constrictive pericarditis with various other aetiologies after inadequate pericardial decortication. ${ }^{28}$

After coronary artery bypass graft surgery radical pericardectomy may be impossible without sacrificing the grafts. Incomplete pericardiectomy, as carried out in our first patient, was adequate, but it would be very difficult to achieve if needed in our second patient. The likely course of events, as constrictive pericarditis develops, suggests the need for early recognition with surgical removal as soon as possible of all intrapericardial blood clots and fluid with as much 
pericardium as can be excised.

\section{CONCLUSIONS}

There is evidence that blood accumulation within the pericardial space plays an important part in the pathophysiology of constrictive pericarditis secondary to cardiac surgery. Possibly with secondary failure of the mechanisms of dissolving blood clots by the pericardial mesothelial cells.

The diagnosis should be considered in patients presenting with right sided heart failure after cardiac surgery. Computed tomography is the best available method of screening postoperatively patients in whom there is a suspicion of developing constriction. Haemodynamic studies will confirm the diagnosis.

Radical pericardiectomy is the treatment of choice. Medical treatment or surgical drainage may, however, partially resolve the constrictive signs when an effusive element is also present in constrictive pericarditis so avoiding the potential risk of graft damage during decortication.

\section{References}

1 Beger HG, Bücherl ES. Perikardektomie nach akuter postoperative Perikarditis: kasuistischer Beitrag. Thoraxchirurgie 1969; 17: 83-7.

2 Marsa R, Mehta S, Willis W, Bailey L. Constrictive pericarditis after myocardial revascularization. Am $\mathcal{J}$ Cardiol 1979; 44: 177-83.

3 Brown DF, Older T. Pericardial constriction as a late complication of coronary bypass surgery. $\mathcal{F}$ Thorac Cardiovasc Surg 1977; 74: 61-4.

4 Rubio PA, Farrell EM, Goens RW. Severe adhesive constrictive pericarditis after coronary artery bypass. South Med 1980; 73: 90.

5 Kutcher MA, King SB III, Alimurung BN, Craver JM, Logue RB. Constrictive pericarditis as a complication of cardiac surgery: recognition of an entity. Am $\mathcal{f}$ Cardiol 1982; 50: 742-8.

6 Sbokos CG, Karayannacos PE, Kontaxis A, Kambylafkas J, Skalkeas GD. Traumatic hemopericardium and chronic constrictive pericarditis. Ann Thorac Surg 1977; 23: 225-9.

7 Glenn EE. Traumatic constrictive pericarditis. Fournal of the Missouri State Medical Association 1940; 37: 7-11.

8 Simon JS, Pluth JR. Constrictive pericarditis. Ann Thorac Surg 1976; 21: 440-1.

9 Cohen MV, Greenberg MA. Constrictive pericarditis; early and late complications of cardiac surgery. Am $\mathcal{F}$ Cardiol 1979; 43: 657-61.
10 Lange RL. Compressive cardiac and circulatory disorders, clinical and laboratory correlation. Am Heart $\mathcal{f}$ 1967; 74: 419-30.

11 Fowler NO. Constrictive pericarditis: new aspects. $A m \mathcal{F}$ Cardiol 1982; 50: 1014-7.

12 Wood P. Chronic constrictive pericarditis. Am $\mathcal{f}$ Cardiol 1961; 7: 48-61.

13 Hancock EW. On the elastic and rigid forms of constrictive pericarditis. Am Heart f 1980; 100: 917-23.

14 Shabetai R, Fowler NO, Guntheroth WG. The hemodynamics of cardiac tamponade and constrictive pericarditis. Am f Cardiol 1970; 26: 480-9.

15 Hirschmann JV. Pericardial constriction. Am Heart $\mathcal{f}$ 1978; 96: 110-22.

16 Rienmuller R, Doppman JL, Reichardt B, Strauer, BE. Diagnostic signs of constrictive pericardial diseases by computed tomography [Abstract]. Fournal of the American College of Cardiology 1983; 1/2: 737.

17 Rotman M, Ratliff NB, Hawley J. Right ventricular infarction; a haemodynamic diagnosis. Br Heart $\mathcal{F}$ 1974; 36: $941-4$.

18 D'Arcy BJ, Gondi B, Nanda NC, Gatewood RP, Biddle T. Real time two-dimensional echocardiography in right ventricular infarction [Abstract]. Am $\mathcal{F}$ Cardiol 1980; 45: 436.

19 Effler DB. Chronic constrictive pericarditis treated with pericardiectomy. Am $\mathcal{f}$ Cardiol 1961; 7: 62-8.

20 Cliff WJ, Grobéty J, Ryan GB. Postoperative pericardial adhesions. F Thorac Cardiovasc Surg 1973; 65: 744-50.

21 Warburg E. Traumatic armour heart. Am Heart $f$ 1955; 49: 633-6.

22 Goldstein S, Yu PN. Constrictive pericarditis after blunt chest trauma. Am Heart $\mathcal{f}$ 1965; 69: 544-50.

23 Porter JM, Ball AP, Silver D. Mesothelial fibrinolysis. $f$ Thorac Cardiovasc Surg 1971; 62: 725-30.

24 Engle MA, Ito T. The postpericardiotomy syndrome. Am f Cardiol 1961; 7: 73-82.

25 Cunningham JN Jr, Spencer FC, Zeff R, Williams CD, Cukingnan $\mathbf{R}$, Mullin $M$. Influence of primary closure of the pericardium after open-heart surgery on the frequency of tamponade, postcardiotomy syndrome, and pulmonary complications. I Thorac Cardiovasc Surg 1975; 70: 119-25.

26 Douglas JS Jr, King SB III, Hatcher CR Jr, Jones EL, Logue RB. Late cardiac tamponade after open heart surgery: a problem of differential diagnosis [Abstract]. Am 7 Cardiol 1975; 35: 133.

27 Beck CS. The effect of surgical solution of chlorinated soda (Dakin's solution) in the pericardial cavity. Arch Surg 1929; 18: 1659-71.

28 Somerville $W$. Constrictive pericarditis with special reference to the change in natural history brought about by surgical intervention. Circulation 1968; 38 (suppl 5): 102-11. 